\title{
The Association between Chronic Conditions, End-of-Life Health Care Use, and Documentation of Advance Care Planning among Patients with Cancer
}

\author{
Cara L. McDermott, PhD, PharmD, ${ }^{1}$ Ruth A. Engelberg, PhD, James Sibley, BS, ${ }^{1,2}$ \\ Mohamed L. Sorror, MD, MSc, ${ }^{3,4}$ and J. Randall Curtis, MD, MPH ${ }^{1}$
}

\begin{abstract}
Background: Multiple chronic conditions (MCCs) are associated with increased intensity of end-of-life (EOL) care, but their effect is not well explored in patients with cancer.

Objective: We examined EOL health care intensity and advance care planning (ACP) documentation to better understand the association between MCCs and these outcomes.

Design: Retrospective cohort study.

Setting/Subjects: Patients aged 18+ years at UW Medicine who died during 2010-2017 with poor prognosis cancer, with or without chronic liver disease, chronic pulmonary disease, coronary artery disease, dementia, diabetes with end-stage organ damage, end-stage renal disease, heart failure, or peripheral vascular disease.

Measurements: ACP documentation 30+ days before death, in-hospital death, and inpatient or intensive care unit (ICU) admission in the last 30 days. We performed logistic regression for outcomes.

Results: Of 15,092 patients with cancer, 10,596 (70\%) had 1+ MCCs (range 1-8). Patients with cancer and heart failure had highest odds of hospitalization (odds ratio [OR] 1.67, 95\% confidence interval [CI] 1.46-1.91), ICU admission (OR 2.06, 95\% CI 1.76-2.41), or in-hospital death (OR 1.62, 95\% CI 1.43-1.84) versus patients with cancer and other conditions. Patients with ACP 30+ days before death had lower odds of in-hospital death (OR 0.65, 95\% CI 0.60-0.71), hospitalization (OR 0.67, 95\% CI 0.61-0.74), or ICU admission (OR 0.71, 95\% CI 0.64-0.80). Conclusions: Patients with ACP 30+ days before death had lower odds of high-intensity EOL care. Further research needs to explore how to best use ACP to ensure patients receive care aligned with patient and family goals for care.
\end{abstract}

Keywords: advance care planning; advance directives; cancer; end-of-life health care; ICU admission

\section{Introduction}

W ITH OUR AGING POPULATION, an increasing number of patients with cancer have multiple chronic conditions (MCCs), or multimorbidity, ${ }^{1,2}$ which can result in higher health care utilization and costs at end of life (EOL) compared to people with no or fewer chronic conditions (CCs). ${ }^{3,4}$ Some patients prefer high-intensity EOL care such as admission to the hospital or intensive care unit (ICU). However, multiple studies have noted that a majority of patients prefer to die outside of the hospital with minimal emergency department (ED) visits and hospitalizations at
EOL. ${ }^{5-7}$ Such high-intensity care may also complicate grief for family members, as avoidance of both ICU admission and in-hospital death is associated with higher quality EOL care assessments among surviving family members. ${ }^{8}$ Previous studies on MCCs in patients with cancer have largely focused on the link between MCCs, subpar chemotherapy receipt, and increased chemotherapy toxicity. ${ }^{9,10}$ While some recent studies have reported an association between the number of CCs and higher risk of hospitalization at EOL, ${ }^{11,12}$ data are limited on how the type and number of MCCs affect health care use among patients with cancer.

\footnotetext{
${ }^{1}$ Department of Medicine, Division of Pulmonary, Critical Care, and Sleep Medicine, Cambia Palliative Care Center of Excellence, University of Washington, Seattle, Washington, USA.

${ }^{2}$ Department of Biobehavioral Nursing and Health Informatics, University of Washington, Seattle, Washington, USA.

${ }^{3}$ Department of Medicine, Division of Medical Oncology, University of Washington, Seattle, Washington, USA.

${ }^{4}$ Clinical Research Division, Fred Hutch, Seattle, Washington, USA.

Accepted February 21, 2020.
} 
Advance care planning (ACP) offers patients the chance to describe their goals for EOL care while they are still able to do so. Completion of ACP can vary with age, race, income, palliative care referral, and type of cancer diagnosis. ${ }^{13-15}$ Effective ACP can be challenging for many reasons. For example, ACP documentation may reflect care and preferences before a cancer diagnosis: in one study $53 \%$ of patients with cancer had completed ACP documentation before receiving a cancer diagnosis. ${ }^{16}$ In a retrospective analysis of patients with advanced cancer, ACP documentation six months before death was associated with reduced hospital admission in the last month of life compared to ACP completed one month before death. ${ }^{17}$ Unfortunately, clinicians may not be aware of prior ACP and thus default to high intensity care and "full code" status. At one center, of patients with advanced cancer admitted to the ICU with a "full code" status, approximately half had a code status change during the admission to do not resuscitate as this was their preference before hospitalization that was not noted during ICU admission. ${ }^{18}$ Finally, changes in advance directives in the last 30 days of life may actually be the outcome of prior high intensity care, making it important to consider the timing of ACP documentation as a predictor of high intensity care. ${ }^{19}$

While the presence of MCCs is becoming more commonplace among patients with cancer and the use of ACP is increasing, ${ }^{20}$ little data are available on the relationship between MCCs and ACP among patients with cancer. Additional data regarding how the number and type of MCCs affect EOL care among people with cancer can help identify populations more likely to be at risk for goal-discordant care and target such patients with interventions to facilitate ACP and receipt of EOL care that is aligned with patient and family goals for care. In this study, we characterized ACP documentation before the last month of life and health care utilization in the last month of life among patients with cancer with or without CCs. We determined the association between number and type of CCs, ACP documentation before the last month of life, and highintensity EOL health care utilization. We also evaluated the relationship between ACP documentation at least 30+ days before death and health care use in the last month of life.

\section{Methods}

\section{Study population}

For this study we utilized the Cambia Metrics database, which links Washington State death certificates to the electronic health record (EHR) of the UW Medicine system. UW Medicine is the largest public health system in the Puget Sound region and includes outpatient clinics, a National Cancer Institute-designated comprehensive cancer center, an academic medical center, two community hospitals, and a county safety-net hospital.

We included patients ages 18 years and up who died between 2010 and 2017 with a diagnosis of poor prognosis cancer (metastatic or aggressive, e.g., acute myeloid leukemia) and at least one of the following CCs identified in the 24 months preceding death: chronic liver disease, chronic pulmonary disease, coronary artery disease, dementia, diabetes with endstage organ damage, end-stage renal disease, heart failure, and peripheral vascular disease. ${ }^{21}$ We adapted Dartmouth Atlas criteria to identify diagnoses based on ICD-9 and ICD-10 codes and to assign patients to UW Medicine facilities, using the criteria of a nonelective nonsurgical hospitalization in the 24 months preceding death or at least two outpatient visits at the same UW Medicine site in the last 32 months of life, with at least one visit in the last 24 months of life. ${ }^{21}$

\section{Covariates}

We identified confounders a priori. Social determinants of health, including race, level of education, and marital status, were identified from the Washington State death certificates. Age, sex, functional limitation, and insurance status were identified from the EHR. We created a binary variable for functional limitation based on codes found in the EHR, drawing from a claims-based algorithm to predict the need for assistance with daily activities of living. ${ }^{22}$ The algorithm uses International Classification of Disease, Healthcare Common Procedure Coding System, and Current Procedural Terminology codes available in patient charts to predict dependency in daily activities. We adjusted for this variable to ensure that associations with CCs were not exclusively due to functional limitations from the CCs.

\section{Outcomes of interest}

Our outcomes of interest were the presence of ACP documentation (which we defined as a health care directive, a durable power of attorney for health care, or a Physician Order for Life-Sustaining Treatments (POLSTs) present in the EHR) at least 30 days before death. We defined high-intensity health care use in the last 30 days of life using previously published criteria, specifically ED visits not resulting in a hospitalization, inpatient hospitalizations, ICU admissions, and in-hospital death. ${ }^{23}$ We identified health care utilization and ACP documentation from the EHR. Place of death (in or out of hospital) was identified from Washington State death certificates.

\section{Statistical analysis}

We described the sample by the number and type of comorbidities. We used multivariable logistic regression to evaluate the relationship between number of comorbidities, type of comorbidities, and outcomes of interest. We controlled for the aforementioned a priori confounders. We also examined the association between ACP documentation 30+ days before death and health care utilization in the last 30 days. Our reference group was patients with cancer and no CCs. Statistical significance was set at $p<0.01$ to account for multiple comparisons.

The University of Washington Institutional Review Board approved this study and issued a waiver of consent to access decedent records, in accordance with Washington State law. We performed all analyses using SAS version 9.4.

\section{Results \\ CCs of interest}

Of 15,092 patients with cancer, 10,596 (70\%) had at least one $\mathrm{CC}$ besides cancer (Table 1). Patients with three or more $\mathrm{CCs}$ were more often older, male, unmarried, non-white, Medicare insured, and with functional limitation compared to those with fewer or no CCs. Of those with three or more noncancer CCs, $84 \%$ had codes indicating functional limitation compared to $43 \%$ of patients with cancer and no other CCs. Among those with two or more chronic noncancer 
Table 1. Demographics and Clinical Condition Prevalence by Number of Multiple Chronic Conditions among 15,092 Patients with Cancer

\begin{tabular}{|c|c|c|c|c|}
\hline & $\begin{array}{c}\text { Cancer and } \\
\text { O MCCs } \\
(\mathrm{n}=4496,30 \%)\end{array}$ & $\begin{array}{c}\text { Cancer and } \\
1 M C C \\
(\mathrm{n}=5687,38 \%)\end{array}$ & $\begin{array}{c}\text { Cancer and } \\
2 M C C s \\
(\mathrm{n}=2680,18 \%)\end{array}$ & $\begin{array}{l}\text { Cancer and } 3 \\
\text { or more MCCs } \\
(\mathrm{n}=2229,15 \%)\end{array}$ \\
\hline Age at death in years, median (range) & $63(18-102)$ & $64(18-101)$ & $67(19-101)$ & $70(20-104)$ \\
\hline Age at death in years, mean (SD) & $62.5(13.4)$ & $63.1(13.6)$ & $66.5(12.9)$ & $69.5(12.1)$ \\
\hline Female, $n(\%)$ & $2212(49.2)$ & $2527(44.4)$ & 1039 (38.8) & $760(34.1)$ \\
\hline Functional limitation, $n(\%)$ & 1944 (43.2) & 3169 (55.7) & $1882(70)$ & $1872(84)$ \\
\hline \multicolumn{5}{|l|}{ Dartmouth MCCs, $n(\%)$} \\
\hline Coronary artery disease & & $367(6)$ & $707(26)$ & $1395(63)$ \\
\hline Dementia & & $75(1)$ & $110(4)$ & $176(8)$ \\
\hline Diabetes & & $183(3)$ & 385 (14) & $754(34)$ \\
\hline Heart failure & & $170(3)$ & 453 (17) & $1161(52)$ \\
\hline Liver disease & & $589(10)$ & $422(16)$ & 417 (19) \\
\hline Peripheral vascular disease & & $100(2)$ & $255(10)$ & $724(32)$ \\
\hline Pulmonary disease & & $586(10)$ & $880(33)$ & $1142(51)$ \\
\hline Renal disease & & $257(5)$ & $529(20)$ & $1130(51)$ \\
\hline \multicolumn{5}{|l|}{ Race, $n(\%)$} \\
\hline White & $3902(87)$ & 4855 (85.5) & $2284(85)$ & $1844(83)$ \\
\hline Asian & $299(6.5)$ & $389(7)$ & $166(6)$ & $145(6.5)$ \\
\hline Black & $165(3.5)$ & $267(5)$ & $147(5)$ & $151(7)$ \\
\hline Other/unknown & $130(3)$ & $176(2.5)$ & $86(4)$ & $89(3.5)$ \\
\hline \multicolumn{5}{|l|}{ Marital status, $n(\%)$} \\
\hline Married or in a partnership & $2741(61)$ & $3261(57)$ & $1459(54)$ & $1184(53)$ \\
\hline Not currently married & $1741(38.5)$ & $2400(42.5)$ & $1202(45)$ & $1022(46)$ \\
\hline Unknown & $14(0.5)$ & $26(0.5)$ & $19(1)$ & $23(1)$ \\
\hline \multicolumn{5}{|l|}{ Education level, $n(\%)$} \\
\hline High school graduate/GED or less & $1580(35)$ & $2119(37)$ & $1625(60)$ & $960(43)$ \\
\hline Some college or more & $2885(64)$ & $3506(62)$ & $1017(39)$ & $1218(55)$ \\
\hline Unknown & $31(1)$ & $62(1)$ & $38(1)$ & $51(2)$ \\
\hline \multicolumn{5}{|l|}{ Insurance type, $n(\%)$} \\
\hline Private & $1972(44)$ & $2295(40)$ & $938(35)$ & $684(31)$ \\
\hline Medicare & $1430(32)$ & $1844(33)$ & $971(36)$ & $897(40)$ \\
\hline Medicaid & 709 (16) & $1102(20)$ & $549(21)$ & $461(21)$ \\
\hline Military, other, or uninsured & $385(8)$ & $446(7)$ & $222(8)$ & $187(8)$ \\
\hline
\end{tabular}

GED, general equivalency diploma; MCCs, multiple chronic conditions; SD, standard deviation.

conditions, the most commonly observed conditions were coronary artery disease, heart failure, chronic pulmonary disease, and renal disease. For those with one noncancer CC, the most common comorbidity was pulmonary disease or liver disease, followed by coronary artery disease.

\section{ACP documentation across all MCCs}

The highest percentage of patients with documentation of $\mathrm{ACP}$ at least 30 days preceding death occurred among those with three or more CCs (34\%) (Fig. 1). For all MCCs, $<35 \%$ of patients had ACP documentation 30+ days before death and $10 \%$ of patients had ACP documentation noted in their EHR for the first time in the last month of life. With respect to type of MCC (Fig. 2), the highest percentage of patients with ACP documentation $30+$ days before death were patients with dementia (35\%), while the lowest was among patients with pulmonary or liver disease, both at $29 \%$. Overall, 9947 (66\%) of the patients with cancer had no ACP documentation in the EHR.

\section{Health care use in last month of life for patients with cancer}

The highest proportion of patients dying in the hospital or dying with an in-patient admission or ICU admission in the last
30 days of life occurred among those with 3+ MCCs (Fig. 1). ED visits (without hospitalization) were slightly more common among those with two MCCs compared to three MCCs ( $4 \%$ vs. $3 \%$ ). Across all categories, $3.7 \%$ of patients visited the ED in their last month of life. Among types of MCCs, $48 \%$ of patients with heart failure died in the hospital compared to $30 \%$ of those with dementia. Patients with cancer and heart failure also had the highest percentage of ICU admission (28\%) and inpatient hospitalization (41\%). The percentage of in-hospital death ranged from $30 \%$ among those with dementia to $48 \%$ among those with heart failure. Patients with heart failure had the highest percentage of hospitalizations in the last 30 days of life $(41 \%)$, while those with chronic pulmonary disease had the lowest (28\%). Patients with heart failure also had the most ICU admissions in the last 30 days (28\%), while those with dementia had the least (14\%).

\section{Odds of outcomes by number and type of MCCs}

Compared to patients with cancer and no other comorbidities, we found significant associations between number of comorbidities and type of comorbidities for EOL health care use and ACP documentation (Table 2). Those with cancer and $3+$ MCCs had the highest odds of inpatient admission, ICU 


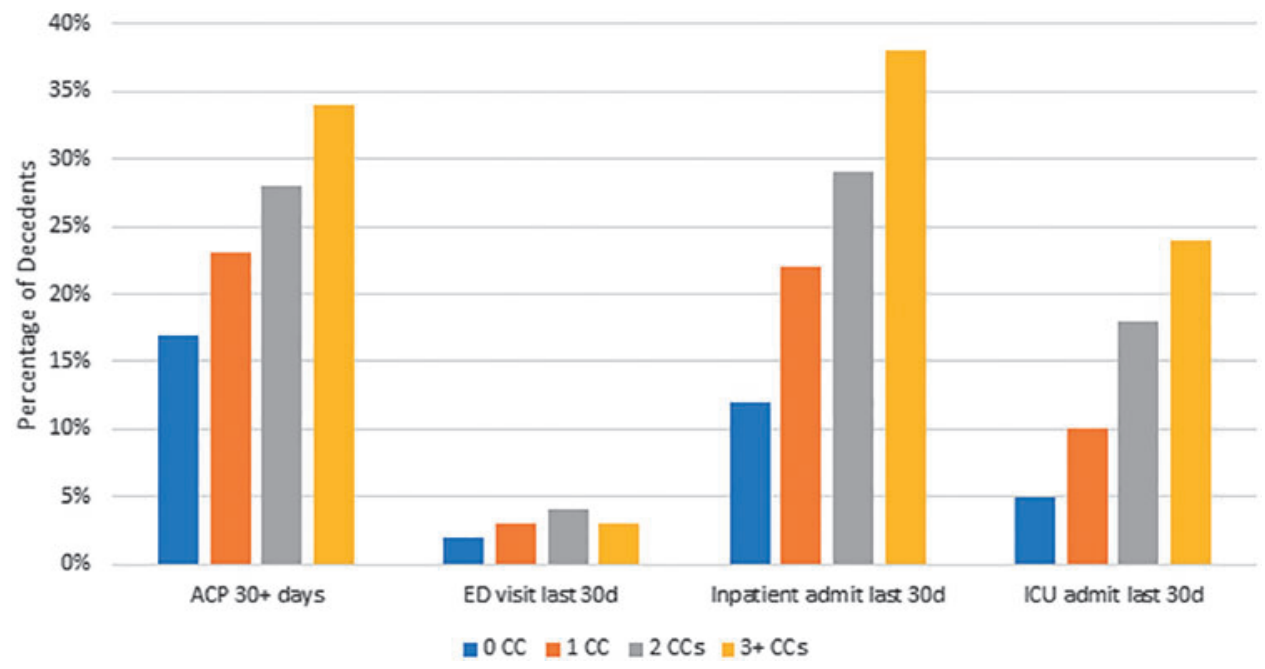

FIG. 1. Outcomes of interest by number of chronic conditions. ACP, advance care planning; CCs, chronic conditions; ED, emergency department; ICU, intensive care unit.

admission, in-hospital death, and ACP 30+ days before death. The highest odds of an ED visit in the last 30 days were among those with cancer and two MCCs rather than those with more MCCs.

Across different types of comorbidity (Table 2), patients with cancer and liver disease had the highest odds of ACP $30+$ days before death (odds ratio [OR] 1.30, 95\% confidence interval [CI] 1.13-1.49). Patients with cancer and heart failure had the highest odds of hospitalization (OR 1.67, 95\% CI 1.46-1.91), ICU admission (OR 2.06, 95\% CI 1.76-2.41), and in-hospital death (OR 1.62, 95\% CI 1.43-1.84).

\section{Association of outcomes and ACP documentation}

Compared to patients without ACP at least 30 days before death, patients with ACP $30+$ days before death had lower odds of in-hospital death (OR $0.65,95 \%$ CI 0.60-0.71), inpatient hospitalization (OR $0.67,95 \%$ CI $0.61-0.74$ ), and
ICU admission (OR $0.71,95 \%$ CI $0.64-0.80$ ). We found no association between ACP 30+ days before death and ED visit without admission (OR 1.19, 95\% CI 0.96-1.48).

\section{Discussion}

We examined the relationship between the number and type of CCs on health care utilization at the EOL and ACP documentation among adults with cancer. We found that utilization of high intensity EOL care increased as the number of CCs co-occurring with cancer increased. We also found that ACP documentation increased as the number of CCs increased, suggesting that patients with cancer and MCCs are more likely to be offered an opportunity to participate in ACP, but the total percent of these patients with numerous and complex conditions (34\%) who complete ACP is still low. We found that patients with ACP documented 30+ days before death were significantly less likely to die in the

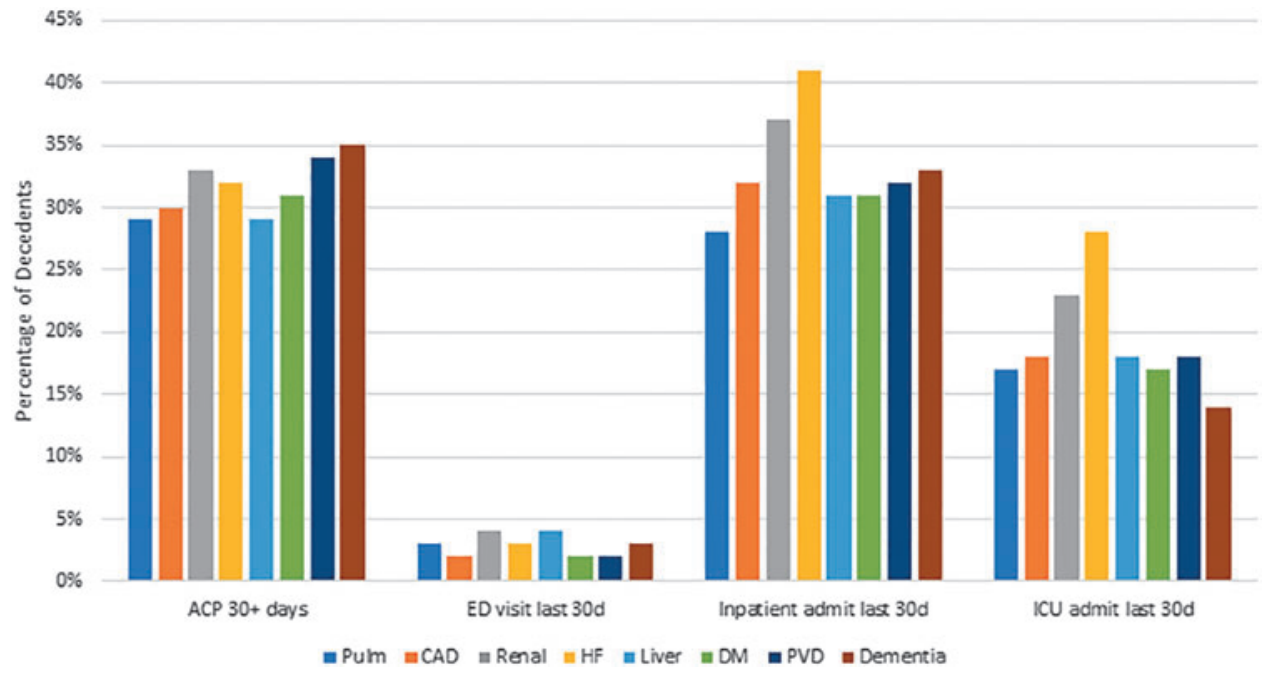

FIG. 2. Outcomes of interest by type of co-occurring chronic conditions. CAD, coronary artery disease; HF, heart failure; DM, diabetes mellitus; PVD, peripheral vascular disease. 


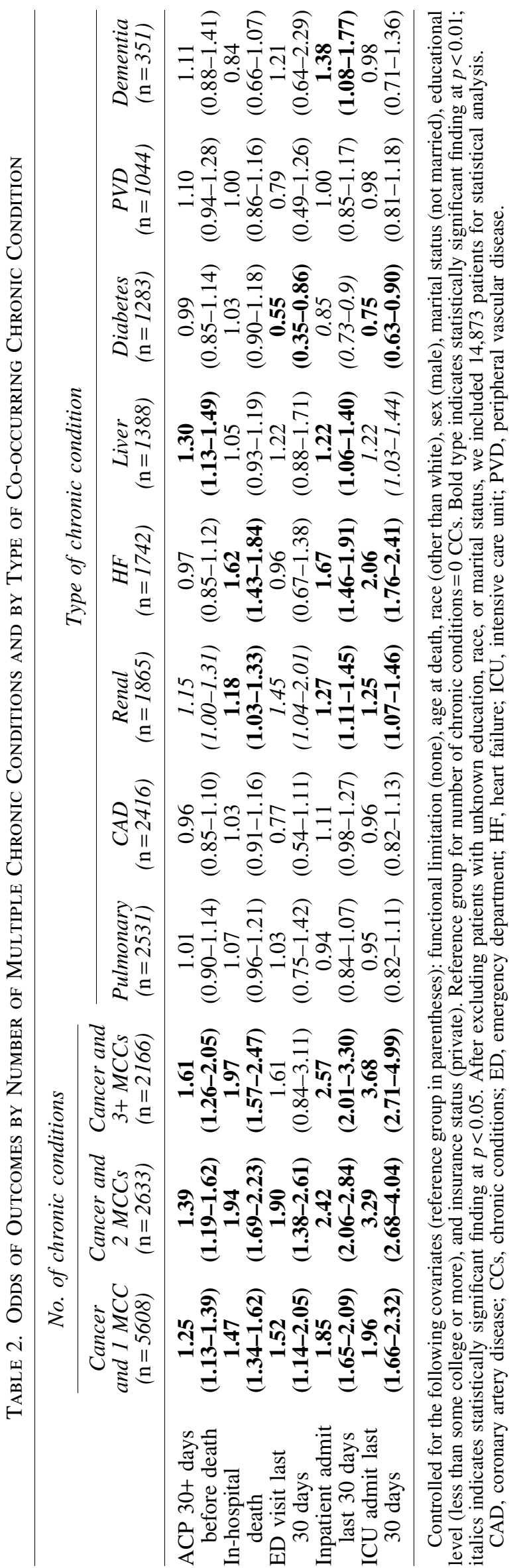

hospital or experience hospitalization or ICU admission in the last 30 days of life, after adjusting for confounders. Among patients with different comorbidities, those with heart failure and cancer had significantly higher odds of inhospital death, as well as hospitalization or ICU admission, in the last 30 days of life.

For this study we utilized the Cambia Metrics database, a database of Washington State death certificates linked to the EHR of UW Medicine. ${ }^{24}$ Unlike previous studies that rely on insurance claims only or focus on Medicare enrollees, we had access to treatment, ACP, and death information for adults ages 18 years and older. This has allowed us to include those under age 65 years who are typically insured privately or through Medicaid. As the average age of comorbidity onset has dropped over time, ${ }^{25}$ our analysis provides additional insight on how to improve EOL care among both older and younger adults with cancer and CCs.

Although other studies have studied MCCs and cancer, they did not evaluate ACP or used smaller sample sizes and could not describe relationships between type of comorbidity and outcomes. For example, Koroukian et al. ${ }^{12}$ used the Health and Retirement Study composed of patient interviews linked to fee-for-service Medicare claims to evaluate EOL health care use and CCs among older adults with cancer. This study was unable to evaluate the association between type of comorbidity and EOL health care use and found no significant association between number of MCCs and health care at EOL, perhaps due to a smaller sample $(n=835) .{ }^{12}$ It is also possible that the severity of MCCs matters more than the number of MCCs, and severity is difficult to estimate without extensive clinical data. Future studies will need to account for the type, combinations, and severity of MCCs.

Similar to a previous study using this database, we found that a higher number of comorbidities was positively associated with higher health care use at EOL. ${ }^{26}$ In addition, we found that patients with the highest comorbidity burden experienced the highest intensity of EOL health care use despite having more ACP documentation in their EHR. This may indicate that patients and families need additional support to provide out of hospital care, especially as death nears. Previous studies found that patients with advanced cancer and their caregivers seek emergency care because of inadequate community-based services, to relieve anxiety and to feel safe in the hospital. ${ }^{27} \mathrm{~A}$ recent analysis noted that commercially insured patients who received ACP also had more hospitalizations ${ }^{28}$; it is possible that in our population sicker patients were more likely to participate in ACP, but also needed hospitalization due to more severe illness.

We found that patients with $\mathrm{ACP}$ at least one month before death received less intensive care. This relationship between ACP and less intensive care was also observed in a study of hematopoietic stem cell transplant recipients ${ }^{29}$ and in a systematic review of ACP in patients with advanced cancer, each demonstrating that ACP $30+$ days before death was associated with significantly lower odds of hospitalization, ICU admission, and death in hospital. ${ }^{30}$

These findings suggest an area for additional palliative care interventions to clarify goals for care and caregiver needs toward the EOL. Such interventions have the potential to spare patients and families from unwanted care and to reduce costs associated with that care. Patients with cancer who had EOL discussions have less intensive health care use 
and lower health care costs in the last week of life compared to patients who did not have such discussions. ${ }^{31}$ May et al. conducted a single-center analysis demonstrating that palliative care is most cost-effective when delivered to patients with cancer and a high comorbidity burden early in the hospital stay, thus avoiding additional laboratory testing and resulting in shorter lengths of stay. ${ }^{32}$ These results were later confirmed in a systematic review and meta-analysis, noting that patients with cancer and comorbidities had significantly lower direct hospital costs after early palliative care consultation. ${ }^{33}$

Our variation in ACP documentation across different types of comorbidities may reflect lack of communication between physicians and patients, but also - for patients with MCCsbetween physicians of different specialties. If a patient has MCCs resulting in a prognosis with a higher degree of uncertainty, physicians often struggle with the timing of ACP conversations. ${ }^{34}$ In this study, we categorized multiple types of documents as ACP. It is possible that the layout and content of some ACP documents (e.g., POLST) more clearly convey patient wishes compared to other documents, for example, a living will. We did not complete a qualitative evaluation of the ACP documents in our database; future research is needed on the most effective type of ACP documentation for goal-concordant care.

Our study is limited by a number of factors. First, we do not have performance status for patients, as these data are not routinely available in the EHR. Second, we used a previously published claims-based analysis to create a variable for functional limitation. As this is a surrogate measure for limitation in activities of daily living (ADLs) rather than a direct measurement of ADLs, it may not accurately depict the impact of functional limitation on patients. Third, we focused on eight specific severe CCs in addition to cancer, but other comorbidities may also be important. However, the comorbidities selected for our analysis represent the most common causes of death in the United States, excluding accidents, infections, and suicide. ${ }^{35}$ Fourth, we sought to capture how the number and type of MCCs may affect health care utilization; future work will need to determine how the severity or various combinations of comorbidities (e.g., heart failure and chronic obstructive pulmonary disease) that accompany a cancer diagnosis affect utilization. Fifth, we identified decedents who had cancer or specific comorbidities, but we are not able to determine the cause of death or the specific disease associated with health care use at the EOL. Finally, as we did not complete a qualitative evaluation of ACP documentation, it is difficult to know the exact wishes of the patients in this study.

In conclusion, we found that an increasing number of $\mathrm{CCs}$ co-occurring with cancer were associated with increased utilization of high intensity care at the EOL. We also found that $\mathrm{ACP}$ documentation increased as the number of $\mathrm{CCs}$ increased, but in adjusted analyses ACP was associated with lower odds of higher intensity care. This research may identify opportunities to explore interventions designed to support oncologists and other specialists (e.g., cardiologists, nephrologists, and pulmonologists) to collaboratively deliver care designed to achieve goal-concordant care at EOL.

\section{Funding Information}

This project is supported by the Palliative Care Research Cooperative Group funded by the National Institute of
Nursing Research U2CNR014637 (pilot grant to C.L.M.). This work was also supported by National Institutes of Health's National Heart, Lung, and Blood Institute Grant K12HL137940 (J.R.C.) and the Cambia Health Foundation (J.R.C.).

\section{Author Disclosure Statement}

No competing financial interests exist.

\section{References}

1. Erdem E: Prevalence of chronic conditions among Medicare Part A beneficiaries in 2008 and 2010: Are Medicare beneficiaries getting sicker? Prev Chronic Dis 2014;11: 130118.

2. Schellevis FG: Epidemiology of multiple chronic conditions: An international perspective. J Comorb 2013;3(Spec. Issue):36-40.

3. Lehnert T, Heider D, Leicht H, et al.: Review: Health care utilization and costs of elderly persons with multiple chronic conditions. Med Care Res Rev 2011;68:387-420.

4. Skinner HG, Coffey R, Jones J, et al.: The effects of multiple chronic conditions on hospitalization costs and utilization for ambulatory care sensitive conditions in the United States: A nationally representative cross-sectional study. BMC Health Serv Res 2016;16:77.

5. Higginson IJ, Daveson BA, Morrison RS, et al.: Social and clinical determinants of preferences and their achievement at the end of life: Prospective cohort study of older adults receiving palliative care in three countries. BMC Geriatr 2017; 17:271.

6. Henson LA, Gao W, Higginson IJ, et al.: Emergency department attendance by patients with cancer in their last month of life: A systematic review and meta-analysis. J Clin Oncol 2015;33:370-376.

7. Alyami HM, Chan RJ, New K: End-of-life care preferences for people with advanced cancer and their families in intensive care units: A systematic review. Support Care Cancer 2019;27:3233-3244.

8. Wright AA, Keating NL, Ayanian JZ, et al.: Family perspectives on aggressive cancer care near the end of life. JAMA 2016;315:284-292.

9. Edwards MJ, Campbell ID, Lawrenson RA, et al.: Influence of comorbidity on chemotherapy use for early breast cancer: Systematic review and meta-analysis. Breast Cancer Res Treat 2017;165:17-39.

10. Hsieh MC, Thompson $\mathrm{T}, \mathrm{Wu} \mathrm{XC}$, et al.: The effect of comorbidity on the use of adjuvant chemotherapy and type of regimen for curatively resected stage III colon cancer patients. Cancer Med 2016;5:871-880.

11. May P, Garrido MM, Aldridge MD, et al.: Prospective Cohort Study of hospitalized adults with advanced cancer: Associations between complications, comorbidity, and utilization. J Hosp Med 2017;12:407-413.

12. Koroukian SM, Schiltz NK, Warner DF, et al.: Social determinants, multimorbidity, and patterns of end-of-life care in older adults dying from cancer. J Geriatr Oncol 2017;8: 117-124.

13. Inoue M: The influence of sociodemographic and psychosocial factors on advance care planning. J Gerontol Soc Work 2016;59:401-422.

14. Huang IA, Neuhaus JM, Chiong W: Racial and ethnic differences in advance directive possession: role of demographic factors, religious affiliation, and personal health 
values in a national survey of older adults. J Palliat Med 2016;19:149-156.

15. Freeman AT, Wood WA, Fox A, et al.: Access to palliative care consultation and advance care planning for adults with high-risk leukemia. J Palliat Med 2018;21:225-228.

16. McDonald JC, du Manoir JM, Kevork N, et al.: Advance directives in patients with advanced cancer receiving active treatment: Attitudes, prevalence, and barriers. Support Care Cancer 2017;25:523-531.

17. Prater LC, Wickizer T, Bower JK, et al.: The impact of advance care planning on end-of-life care: do the type and timing make a difference for patients with advanced cancer referred to hospice? Am J Hosp Palliat Care 2019;36:10891095.

18. El-Jawahri A, Lau-Min K, Nipp RD, et al.: Processes of code status transitions in hospitalized patients with advanced cancer. Cancer 2017;123:4895-4902.

19. Enguidanos S, Ailshire J: Timing of advance directive completion and relationship to care preferences. J Pain Symptom Manage 2017;53:49-56.

20. Portanova J, Ailshire J, Perez C, et al.: Ethnic differences in advance directive completion and care preferences: What has changed in a decade? J Am Geriatr Soc 2017;65:13521357.

21. Iezzoni LI, Heeren T, Foley SM, et al.: Chronic conditions and risk of in-hospital death. Health Serv Res 1994;29:435460.

22. Faurot KR, Jonsson Funk M, Pate V, et al.: Using claims data to predict dependency in activities of daily living as a proxy for frailty. Pharmacoepidemiol Drug Saf 2015;24: 59-66.

23. Earle CC, Neville BA, Landrum MB, et al.: Evaluating claims-based indicators of the intensity of end-of-life cancer care. Int J Qual Health Care 2005;17:505-509.

24. Curtis JR, Sathitratanacheewin S, Starks H, et al.: Using electronic health records for quality measurement and accountability in care of the seriously ill: Opportunities and challenges. J Palliat Med 2018;21(S2):S52-S60.

25. Akushevich I, Kravchenko J, Ukraintseva S, et al.: Time trends of incidence of age-associated diseases in the US elderly population: Medicare-based analysis. Age Ageing 2013;42:494-500.

26. Wagner E, Patrick DL, Khandelwal N, et al.: The influence of multimorbidity on health care utilization at the end of life for patients with chronic conditions. J Palliat Med 2019;22:1260-1265.
27. Henson LA, Higginson IJ, Daveson BA, et al.: 'I'll be in a safe place': A qualitative study of the decisions taken by people with advanced cancer to seek emergency department care. BMJ Open 2016;6:e012134.

28. Ashana DC, Chen X, Agiro A, et al.: Advance care planning claims and health care utilization among seriously ill patients near the end of life. JAMA Netw Open 2019;2: e1914471.

29. Cappell K, Sundaram V, Park A, et al.: Advance directive utilization is associated with less aggressive end-of-life care in patients undergoing allogeneic hematopoietic cell transplantation. Biol Blood Marrow Transplant 2018;24: 1035-1040.

30. Starr LT, Ulrich CM, Corey KL, et al.: Associations among end-of-life discussions, health-care utilization, and costs in persons with advanced cancer: A systematic review. Am J Hosp Palliat Care 2019;36:913-926.

31. Zhang B, Wright AA, Huskamp HA, et al.: Health care costs in the last week of life: Associations with end-of-life conversations. Arch Intern Med 2009;169:480-488.

32. May P, Garrido MM, Cassel JB, et al.: Palliative care teams' cost-saving effect is larger for cancer patients with higher numbers of comorbidities. Health Aff (Millwood) 2016;35:44-53.

33. May P, Normand C, Cassel JB, et al.: Economics of palliative care for hospitalized adults with serious illness: A meta-analysis. JAMA Intern Med 2018;178:820-829.

34. Schonfeld TL, Stevens EA, Lampman MA, et al.: Assessing challenges in end-of-life conversations with elderly patients with multiple morbidities. Am J Hosp Palliat Care 2012;29:260-267.

35. Heron M: Deaths: Leading causes for 2016. Natl Vital Stat Rep 2018;67:1-77.

Address correspondence to: Cara L. McDermott, PhD, PharmD Department of Medicine

Division of Pulmonary, Critical Care, and Sleep Medicine Cambia Palliative Care Center of Excellence University of Washington 325 Ninth Avenue, Box 359765 Seattle, WA 98104 USA

E-mail: clm2@uw.edu 